\title{
The medicalization of disease as a factor of abandonment and dissatisfaction with HIV treatment services
}

\author{
Carmen Rodríguez Reinado*, Teresa Blasco, Hernández Jesus, Nzang Esono \\ From 17th International Symposium on HIV and Emerging Infectious Diseases (ISHEID) \\ Marseille, France. 23-25 May 2012
}

\section{Introduction}

Medicalization of the disease is a social phenomenon not exclusive of industrialized societies. In countries with medium and low human development indexes and developing economies, this process is prevailing due to the expansion of the biomedical model. This is the case of many African countries and more concretely in Equatorial Guinea.

\section{Methodology}

Qualitative research, based on the application of semistructured interview (30) as the technique for gathering information. Ambit: Bata, Equatorial Guinea. Purposeful sampling: homogeneous type by subgroup; 1. HIV-positive people who abandoned diagnostic and treatment services. 2. HIV-positive people who are utilising HIV treatment services. Location: General Hospital and Outpatient Treatment Centers. Unit of analysis: utilization of diagnostic and treatment services.

\section{Method of analysis}

Grounded Theory Method. Data triangulation: internal by two observers and theoretical.

\section{Results}

- Drugs are a central topic on the discourse about the disease.

- Regardless of the population profile, all the informants share a medicalized view of HIV and its treatment

- In the asymptomatic phase of HIV, avoiding to prescribe drugs reinforces the process of non-acceptance of the disease leading the patient to question his positive diagnosis.
- Within the profile of HIV-positive respondents still in treatment, medication is the element of the care process that takes on more importance. Avoiding to prescribe drugs is an element of dissatisfaction with health services.

- In the profile of HIV-positive respondents who abandoned HIV diagnostic and treatment services, no prescription drugs was one of the reasons for abandonment.

\section{Conclusions}

The medicalization of the disease has affected the social construction of HIV as a disease and is one of the reasons for abandonment and dissatisfaction with the care received in diagnostic and treatment centers. It therefore represents a factor for intervention and to modify in order to reduce the rates of abandonment for such services.

Published: 25 May 2012

doi:10.1186/1742-4690-9-S1-P91

Cite this article as: Reinado et al:: The medicalization of disease as a factor of abandonment and dissatisfaction with HIV treatment services. Retrovirology 2012 9(Suppl 1):P91.

* Correspondence: carmenrr1974@yahoo.es

University of Huelva, Huelva, Spain

(c) 2012 Reinado et al; licensee BioMed Central Ltd. This is an Open Access article distributed under the terms of the Creative Commons 